\title{
Vincent Petit, Catholiques et Comtois. Liturgie diocésaine et identité régionale au $\mathrm{XIX}^{\mathrm{e}}$ siècle
}

Cartes, graphiques, notes bibliographiques, index, cahier photo Paris, Les Éditions du Cerf, coll. « Histoire religieuse de la France », 36, 2011, 720 p.

\section{Paul Airiau}

\section{(2) OpenEdition}

\section{Journals}

Édition électronique

URL : http://journals.openedition.org/assr/25592

DOI : $10.4000 /$ assr.25592

ISSN : $1777-5825$

Éditeur

Éditions de l'EHESS

Édition imprimée

Date de publication : 30 décembre 2013

Pagination : 270

ISSN : 0335-5985

\section{Référence électronique}

Paul Airiau, «Vincent Petit, Catholiques et Comtois. Liturgie diocésaine et identité régionale au xix ${ }^{\mathrm{e}}$ siècle ", Archives de sciences sociales des religions [En ligne], 164 | 2013, mis en ligne le 13 mars 2014, consulté le 21 septembre 2020. URL : http://journals.openedition.org/assr/25592 ; DOI : https:// doi.org/10.4000/assr.25592

Ce document a été généré automatiquement le 21 septembre 2020

(c) Archives de sciences sociales des religions 


\section{Vincent Petit, Catholiques et} Comtois. Liturgie diocésaine et identité régionale $\mathrm{au} \mathrm{XIX}^{\mathrm{e}}$ siècle

Cartes, graphiques, notes bibliographiques, index, cahier photo Paris, Les Éditions du Cerf, coll. « Histoire religieuse de la France », 36, 2011, $720 \mathrm{p}$.

Paul Airiau

\section{RÉFÉRENCE}

Vincent Petit, Catholiques et Comtois. Liturgie diocésaine et identité régionale au xIX ${ }^{\mathrm{e}}$ siècle, Cartes, graphiques, notes bibliographiques, index, cahier photo, Paris, Les Éditions du Cerf, coll. « Histoire religieuse de la France », 36, 2011, 720 p. 
1 La liturgie est une affaire trop sérieuse pour être laissée aux mains des liturges. À cette affirmation, tout historien ne saurait que souscrire. En effet, c'est peu dire qu'il nous manque encore en France une histoire de la liturgie catholique des $\mathrm{XIX}^{\mathrm{e}}$ et $\mathrm{xx}^{\mathrm{e}}$ siècles digne de ce nom - une histoire qui ne soit pas sainte, mais universitaire, sécularisée, dépassionnée, désacralisée, profane. Aussi faut-il se réjouir que, après Xavier Bisaro qui revisitait l'histoire des liturgies du $\mathrm{XVIII}^{\mathrm{e}}$ siècle en se penchant sur la liturgie parisienne (Une nation de fidèles. L'Église et la liturgie parisienne au XVIII siècle, Tournai, Brepols, 2006 - recension par Daniel-Odon Hurel, ASSR, 142), Vincent Petit explore la place de la liturgie dans le catholicisme du XIX ${ }^{\mathrm{e}}$ siècle.

Il avait déjà proposé un cadre interprétatif général dans Église et Nation. La question liturgique en France au XIX siècle (Rennes, Presses universitaires de Rennes, 2010), partie de sa thèse dont le présent ouvrage, bien plus gros, est le complément nécessaire. En effet, après avoir observé la reconfiguration de la liturgie catholique dans un sens intransigeant, centralisateur et unificateur, destiné à permettre à l'Église de faire pièce à une nation démocratique en construction, il déplace l'observation sur un cas particulier, la Franche-Comté. L'objet est particulièrement bienvenu dans la perspective de son projet global visant à comprendre la constitution en des temps démocratiques de communautés culturelles disposant de leurs propres références (Églises, groupes religieux, provinces, pays, départements...), mais ayant toujours à s'inscrire dans une relation complexe avec d'autres communautés relevant d'échelles différentes (nation, Église universelle...). En effet, la Franche-Comté, province viscéralement patriote, mais de tradition romaine dans l'espace français gallican, fut l'avant-dernier diocèse français à adopter la liturgie romaine, en 1874, manifestant ainsi une certaine réticence à la romanisation comprise comme «intransigification" (on m'excusera, je l'espère, ce néologisme) associant centralisation, uniformisation et résistance au libéralisme. L'objectif est donc de comprendre comment ce paradoxe se construit puis se résout, et comment il contribue à la construction d'une identité régionale. Bref, Besançon, Rome et Paris, tel est le triangle de l'étude.

3

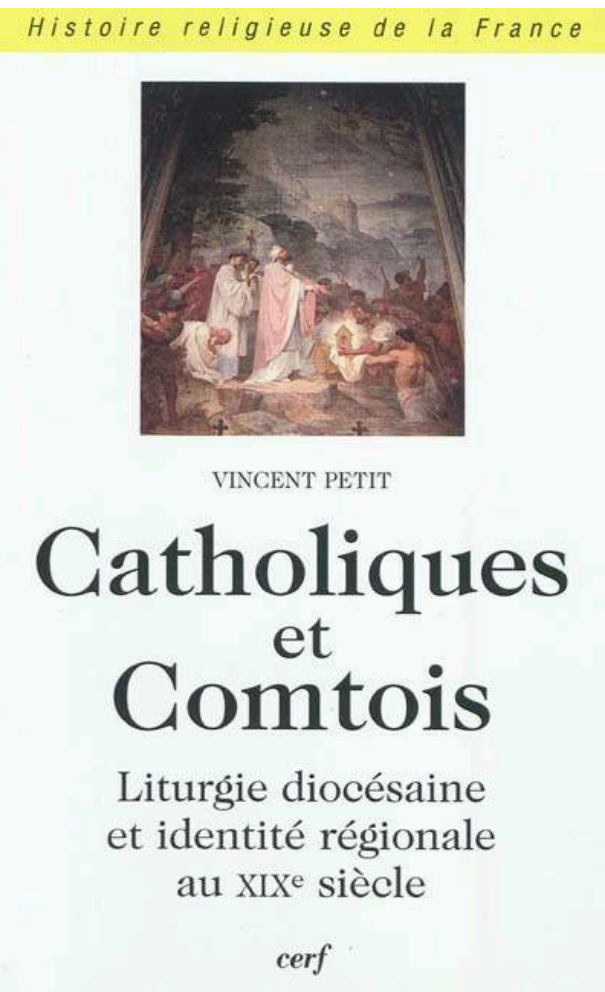


ses prêtres historiens du diocèse de Dijon). L'intérêt de l'approche de l'auteur est de mettre en avant combien la fracture révolutionnaire, dans le contexte spécifique de la Franche-Comté, joue, par le biais de réappropriations et réinterprétations successives, jusqu'à la fin des années 1870, en se focalisant finalement sur la liturgie. Réduction à merci du corps ecclésiastique après l'épiscopat du gallican et bonapartiste Le Coz, inculturation mennaisienne du jeune clergé dans les années 1820-1840 (avec les futurs évêques Gerbet, Gousset et Doney), construction d'un groupe clérical mal contrôlé par une faible administration épiscopale (pour des raisons purement circonstancielles, la succession rapide des titulaires du siège : quatre de 1815 à 1834), nomination d'un jeune évêque autoritaire formé à Saint-Sulpice et "plus gallican en pratique qu'idéologue " (p.137), le diocèse de Besançon voit se mettre en place les éléments de la querelle liturgique qui va le déchirer sous son nouvel archevêque.

L'articulation de plusieurs strates d'explication fait tout l'intérêt de cette partie: le bouleversement révolutionnaire et sa réactivation régulière à travers diverses questions en ligne de fond; un double phénomène générationnel, le romantisme catholique sous sa forme mennaisienne et la reconstruction cléricale d'après la Révolution; l'importance des circonstances et des personnalités à travers les successions épiscopales. Vincent Petit articule ainsi histoire bataille et histoire de longue durée, pour reprendre des catégories banales, mais en montrant combien les individus, tout particuliers qu'ils soient, sont toujours pris dans des structures qui les marquent et déterminent en partie non seulement ce qu'ils sont, mais aussi ce qu'on veut qu'ils soient ou qu'on dit qu'ils sont - Mgr Mathieu est ainsi nécessairement, pour ses opposants bisontins, un gallican dans le sens le plus détestable qu'il soit: cryptohérétique, autoritaire, administratif.

5 La deuxième partie, "Dispute d'état et controverse publique : la querelle liturgique ", se penche en dix chapitres sur les vingt années du conflit autour de la liturgie dans le diocèse bisontin. Le mouvement de romanisation n'est pas sans trouver des défenseurs, qui, en leur particulier, utilisent exclusivement le rite romain, mais tardivement (à partir du début des années 1850). Surtout, ces défenseurs sont partie prenante de réseaux romains déjà constitués qui vont jouer dans les tensions internes à Besançon un rôle non négligeable. Or, ces réseaux sont associés à des pratiques d'influence: médiatisation, circulation d'informations par relations épistolaires, utilisation des relations liées à la sociabilité cléricale... Face à eux, Mgr Mathieu entend conserver son autorité épiscopale tant contre l'intervention directe de Rome que contre les romanisateurs, sans être pour autant gallican en liturgie, et maintenir un certain nombre d'usages bisontins qu'il estime parfaitement justifiés. Cette situation et ces volontés épiscopales expliquent assez largement le développement et les rebondissements de la querelle. Mgr Mathieu s'engage dans la validation romaine d'une liturgie bisontine. Se lançant dans une enquête érudite dont il tire un mémoire adressé à la Congrégation des Rites en 1855, il demande la possibilité de reprendre bréviaire et missel de la fin du XVII siècle. Ce choix permet d'articuler particularismes locaux, formes romaines et héritage tridentin. Mais sa demande est refusée en 1856. L'archevêque s'oriente alors vers la construction d'un propre diocésain "romanocompatible » pour lequel il obtient un indult en 1862. Il faudra cependant plus de dix ans pour que la réforme entre en application, puisque Mgr Mathieu, au nom des difficultés à changer des usages fortement enracinés, se hâte fort lentement - l'édition des nouveaux livres prend notamment beaucoup de temps - s'appuyant sur les difficultés du contexte politique pour tergiverser davantage encore. Entre-temps, il a 
dû faire face, de 1856 à 1865 , à une très forte polémique diocésaine menée par des clercs romanisateurs, intransigeants et donnant vite un tour personnel à la querelle : les abbés Maire, Mercier, Bergier et le chanoine Thiébaud (trois sont déliés du ministère paroissial et disposent ainsi d'une certaine autonomie). Arguments théologiques, historiques, pratiques, tout est bon pour contester les décisions épiscopales et leurs orientations : gallicanisme larvé contre intransigeance romaine, particularisme régional contre unité universelle, lenteur hypocrite contre ardeur enthousiaste, édition locale coûteuse contre publication industrielle bon marché.

À cette exposition chronothématique de la querelle, Vincent Petit associe une sociohistoire de l'échec final de Mgr Mathieu. Il suit au plus près la constitution, au sein du clergé bisontin, de clivages géographiques (conférences ecclésiastiques), générationnels (les plus jeunes sont plus romains), professionnels (le chapitre, les séminaires, la mission diocésaine sont dominés par les romains) autour de la question liturgique. Ils aboutissent à une confusion pratique qui n'est pas sans répercussions visibles (différences d'ornements, de calendrier, etc.), sans compter les répercussions dans le fonctionnement diocésain. Les carrières dépendent de la position prise dans la querelle, les institutions diocésaines se grippent, les séminaristes sont l'objet de pressions. In fine, quoique le cardinal Mathieu défende la souveraineté temporelle du pape et appuie le syllabus, les "arguments des partisans de l'unité liturgique [...] se muent en doctrine cohérente et agressive, tournée contre le catholicisme libéral et le gallicanisme érigé en hérésie, celle des "libéraux bisontins" » (p. 385). Ainsi, la situation locale est lue à l'aune de la situation universelle, et réciproquement - le tout au service d'intérêts particuliers, ceux des opposants à l'archevêque. L'interaction des échelles est systématique, perdurant jusqu'après la mort du cardinal Mathieu et pesant sur la promotion à l'épiscopat de l'abbé Besson, son ancien collaborateur.

7 De cette partie peuvent être tirées des réflexions nombreuses et variées. Je n'en avancerai ici qu'une seule. Les acteurs de la querelle y ont apporté des explications différentes, selon la position qu'ils y occupaient. Le cardinal Mathieu tend à imputer l'ardeur romaine de ses opposants à leur caractère (p. 253-256), alors que ceux-ci font primer des considérations idéologiques (p. 258-268). Tout compte fait, on retrouverait les prodromes des analyses du P. Yves Congar sur l'intégrisme («Intégrisme et mentalité de droite", La Vie intellectuelle, 1950), qui insistait sur la structuration psychologique particulière caractérisant un intégrisme qu'il étendait rétrospectivement jusqu'au milieu du $\mathrm{XIX}^{\mathrm{e}}$ siècle pour y inclure ce qu'on a fini par appeler l'intransigeance. Valorisant le poids du caractère dans l'histoire, le dominicain entendait bien sûr dévaloriser par ce biais ses opposants, mais s'interrogeait aussi en même temps sur la manière d'expliquer en histoire: comment tenir ensemble l'importance des facteurs humains et des conditionnements sociaux, sans verser dans une histoire psychologisante ou tout faire dépendre des structures. À ces questions, les fines analyses de l'auteur, conduites au niveau microscopique, permettent d'apporter des éléments de réponse. Dépassant la simple histoire locale ou érudite, il accède au niveau macroscopique en articulant en fait deux niveaux : l'individualité et l'individu. Il donne à réfléchir sur l'individualité de l'individu. En effet, il montre parfaitement comment, chez tous les acteurs de la querelle, interagissent en permanence, les habitus d'état, propres au monde clérical, les particularités personnelles, notamment de caractère, et les circonstances. La dimension proprement sociale de l'individu, la dimension irréductible de la personne, les conditions qui amènent à les faire jouer sont ici associées de manière fort heureuse. Cela permet de tenir ensemble une analyse 
sociohistorique de fond (les logiques idéologiques à l'œuvre: romanisation, intransigeance romaine, gallicanisme...), une histoire psychologique d'acteurs en interrelation permanente (les traits de caractère: emportement, passion, raideur, certitude de sa valeur...) et le déroulé chronologique des désaccords. Ainsi, le chanoine Thiébaud apparaît comme un Romain passionné violemment hostile à son archevêque, ou comme animé d'une passion romaine tournée contre son hiérarque, de 1845 à 1884 soit même après la mort du cardinal. La situation sociale (un chanoine), les choix idéologiques (l'intransigeance), le caractère (l'assurance acerbe), la factualité (les choix de Mgr Mathieu, les décisions romaines...) interagissent en permanence.

In fine, on ne peut s'empêcher de s'interroger sur la passion intransigeante. Indéniablement, l'intransigeance française (mais elle n'est sans doute pas la seule) a été une pensée foncièrement polémique, dans sa constitution (contre le libéralisme) et dans son fonctionnement (par la dénonciation virulente d'ennemis), au moins jusque dans les années 1870 en ce qui concerne les affaires internes au catholicisme (mais qui sont toujours plus ou moins reliées aux affaires extraecclésiales). Aussi Vincent Petit peut-il la rapprocher de "réactions césaroplébiscitaires » (p. 406). Il y aurait à creuser cette ligne d'analyse, ouverte par Philippe Boutry dans Prêtres et paroisses au pays du curé d'Ars (1986), lorsqu'il montrait l'arrivée dans les années 1850-1860 d'une génération de prêtres populaires affirmant leur autorité face au monde nobiliaire ou notabiliaire. Le parti intransigeant pourrait utilement être rapproché de ces autres partis, qui, plus tard dans l'histoire de France, eurent une fonction tribunitienne. Même fonction de contestation, même obsession unitaire autour d'un centre détenteur de vérité, même fonctionnement par exclusion. À moins qu'il ne faille faire le rapprochement avec cette forme particulière de démocratie qui fut sous la Révolution, celle qui affirme l'unicité du demos composé de membres égaux communiant dans une même volonté en rejetant radicalement tous ceux qui semblent porter atteinte à l'indivisibilité civique. L'intransigeance polémique serait ainsi la forme catholique de la passion démocratique moderne - et j'ajouterais même, dans sa version cléricale, une virilité de substitution: la polémique remplace la violence physique, ce propre viril (théoriquement) interdit au prêtre.

9 La troisième et dernière partie, "Après la Révolution: l'affirmation d'une identité catholique régionale ", reprend l'ensemble du dossier en étudiant la création au XIX ${ }^{\mathrm{e}}$ siècle d'une identité régionale comtoise. Huit chapitres vont ainsi se pencher sur l'interaction complexe entre particularité comtoise, spécificité cléricale et identité liturgique. Dans tous les cas, l'auteur montre parfaitement comment fonctionne l'invention des traditions, et ce spécialement au niveau ecclésiastique. L'investissement de l'érudition historique par le clergé bisontin est un phénomène qui se retrouve dans d'autres diocèses (on a déjà mentionné S. Milbach, on peut citer aussi François Ploux, "Les curés historiens de village et les tentatives de restauration de l'autorité cléricale après la Révolution ", Le Mouvement Social 2008/3, 224, p. 21-33). Elle s'explique par la fonction sociale acquise par l'histoire dans la société française, mais également par l'influence mennaisienne sur l'apologétique catholique. L'histoire sert de preuve et d'argument d'authenticité, de validité, de vérité. Elle est intrinsèquement liée à la dispute liturgique et devient même bientôt un instrument de dispute d'état, en ce sens qu'elle permet l'expression des conflits au sein du monde clérical. La dispute liturgique transparaît dans la pratique historienne des ecclésiastiques qui relisent l'histoire du diocèse de Besançon à l'aune de leur querelle - rapport à l'apostolicité, au siège romain, au Concile de Trente, au jansénisme... Même, les nécrologies, cette forme particulière 
d'histoire, jouent ici un rôle fondamental dans l'expression de la conflictualité, car ces figures imposées n'empêchent pas la manifestation d'appréciations personnelles ou de relectures idéologiques : « Elles sont [...] avant tout un exercice de confraternité, propre à l'état ecclésiastique, dont elles reprennent les codes mais aussi les clivages» (p. 514). Développement d'une compétence de spécialistes des traditions, puis développement d'une histoire idéologisée donc. Enfin, développement d'une justification des changements, des permanences ou des restaurations : le corps clérical appuyé sur sa nouvelle érudition mise au service d'un projet idéologique développe des choix pastoraux en relation avec ses objectifs. Ainsi le cardinal Mathieu entend-il conforter par l'histoire le relatif particularisme bisontin alors que s'imposent l'uniformisation liturgique et l'italianisation de la piété. Reliques, saints, dévotions anciennes sont promus. Mais dans tous les cas, Romains et Bisontins doivent se confronter à la critique historique, dont les règles et les effets sont les mêmes pour tous. Cependant, les premiers s'en sortent bien mieux que les seconds, car pour eux «la liturgie relève d'enjeux ecclésiaux et dogmatiques et d'une ambition englobante, celle d'une nouvelle sacralisation ». Aussi la " défense des rites propres au diocèse ne pouvait qu'échouer » (p. 609).

10 Vincent Petit aboutit ainsi à la fort utile distinction entre une "romanité historique » et une "romanité ecclésiologique considérée comme nouvelle». Il reprend in fine le point de départ de son travail en montrant, en quelques mots, combien la configuration des $\mathrm{XVI}^{\mathrm{e}}$-XVIII ${ }^{\mathrm{e}}$ siècles s'est transformée. En conservant, ou en tentant de conserver, la situation ancienne, celle d'un décalage particulariste au sein du royaume de France exprimé par la liturgie, Besançon aboutit en fait dans les années 1850 à un décalage liturgique au sein du catholicisme français que certains de ses clercs tentent de justifier au nom du particularisme régional historique. Mais ils ne perçoivent pas que ce décalage liturgique devient dissonance ecclésiologique aux yeux de leurs adversaires locaux et nationaux, mais aussi pour le centre romain auquel ils se veulent pourtant fidèlement attachés au nom des anciennes traditions locales. On voit combien le cardinal Mathieu n'a pas compris le changement qui, entre 1750 et 1850, transforme l'articulation des diocèses à Rome, des évêques au pape entouré de la Curie.

Le processus d'uniformisation du catholicisme que fut la romanisation intransigeante ne peut ici qu'être mis en parallèle avec l'uniformisation nationale qui s'accentuera dans les années 1880-1900. Que celui-là se soit achevé avant celle-ci explique sans doute pourquoi le catholicisme a pu résister à la vague anticléricale de la République installée, mais aussi pourquoi la République fut anticléricale (les républicains se constituèrent comme anticléricaux précisément lorsque l'imposition de l'ecclésiologie intransigeante triompha) et pourquoi le conflit fut si virulent. Bref, l'intransigification alimentait les conflits auxquels elle dut faire face, en même temps qu'elle permettait d'y faire face. Cette uniformisation catholique manifestait sa capacité d'intégration et d'accueil relatif des pluralités locales, d'où elle tirait sa force. Les diocèses articulaient spécificités régionales et universalité romaine dans un cadre que les réalités politiques, administratives et juridiques imposaient de considérer comme national. Ne retrouve-ton pas là ce que sut faire également la République, construisant des petites nations au sein de la grande nation? Et n'a-t-on pas là une des clés de compréhension du catholicisme du XIX ${ }^{e}$ siècle : l'articulation d'une double, voire d'une triple fidélité, à la région, à la nation, à l'Église ? Le parallèle avec cette autre organisation transnationale 
que fut le Parti communiste peut ici être fait et il serait sans doute opportun, un jour, de mener une étude croisée des modalités catholique et communiste d'être français.

L'auteur invite ainsi à réfléchir sur les niveaux d'appartenance des individus et sur la capacité des systèmes idéologiques à les prendre en compte pour être efficaces. Nul doute que l'on saisira l'utilité de ces pistes après que Vatican II a partiellement rebattu les cartes de l'uniformisation liturgique, et que les décisions de Benoît XVI ont, depuis quelque peu, de nouveau modifié la donne. Mais l'on observera ici aussi les changements de configuration: les logiques territoriales cèdent désormais le pas à des logiques culturelles déterritorialisées. Le choix de la liturgie de 1962 ou de celle de 1969-1970, en partie en lien avec l'ecclésiologie et l'histoire internationale et nationale qui l'accompagnent, relève de choix individuels d'inscription dans une culture en partie recomposée, ce que nombre de liturges n'ont pas compris. On ne peut finalement s'empêcher de penser qu'il y aurait donc une utilité para-théologique de la pratique historique : poser à nouveau frais des questions que les habitus professionnels tendent à régler trop rapidement comme lors des querelles du $\mathrm{xIX}^{\mathrm{e}}$ siècle, par la voie de l'imposition implicitement autoritaire, de la dépréciation plus ou moins méprisante, voire de la polémique. Bref, c'est peu dire que le livre de Vincent Petit est utile, tant il donne l'occasion de penser. 